International Journal of Wireless \& Mobile Networks (IJWMN) Vol. 3, No. 4, August 2011

\title{
PROBABILISTIC ROUTING USING QUEUING THEORY FOR MANETs
}

\author{
Gaurav Khandelwal, Giridhar Prasanna, Chittaranjan Hota \\ Birla Institute of Technology \& Science-Pilani \\ Hyderabad Campus, Hyderabad, INDIA \\ \{gaurav, prasanna, hota\} @bits-hyderabad.ac.in
}

\begin{abstract}
Mobile ad-hoc networks pose real difficulty in finding the multihop shortest paths because of continuous changing positions of the nodes. Traditional ad-hoc routing protocols are proposed to find multi-hop routes based on shortest path routing algorithms, which cannot effectively adapt to time-varying radio links and network topologies of Ad-hoc networks. In this paper we proposed an enhanced routing algorithm, which uses probabilistic approach for the stability of the neighboring nodes in finding and maintaining the routing paths in Ad-hoc networks. The probability of a node being stable in the path is modeled by queuing theory, where the stability of a node is measured by number of packets arrived at a node and the number of packets being serviced by the node per unit time. Proposed approach shows significant improvement over the traditional Ad-hoc on-demand distance vector routing protocol as analyzed in the result analysis section.
\end{abstract}

\section{Keywords}

AODV, MANET, Probabilistic routing, Stability, Queuing theory.

\section{INTRODUCTION}

An Ad-hoc wireless network consists of a group of mobile nodes which communicate with each other through the wireless links in a distributed fashion without a centralized controller. Mobile Ad-hoc Networks (MANETs) are autonomous collection of mobile nodes. MANET usually lacks any type of fixed infrastructure. As nodes in MANET are mobile, so the link failure and re-establishment of routes takes place frequently. It is difficult to keep track of all these nodes and their locality centrally. So it is essential to find out the geographic location of nodes only when communication channel has to be established, in this wireless environment wherein bandwidth available for the communication is constrained. Moreover, these nodes keep moving with different speed and power is an important constraint for them all the times. A topology of 18 nodes Ad-hoc network is shown in figure 1. Because the nodes are mobile, the topology keeps changing which makes the transfer of packets complicated.

To discover and maintain the routes in ad-hoc networks requires more control traffic which makes the task of ad-hoc routing more complex and less efficient. Several researches on developing ad-hoc routing protocols for MANETs have been proposed [1,5]. These routing protocols can be classified as proactive protocols [1, 2], reactive protocols [4], or hybrid of the both [5].Pro-active (table-driven) routing protocols maintain fresh lists of destinations and their routes by periodically distributing routing tables throughout the network. The main

DOI : $10.5121 /$ ijwmn.2011.3410 
International Journal of Wireless \& Mobile Networks (IJWMN) Vol. 3, No. 4, August 2011

disadvantages of such algorithms are the respective amount of data for maintenance and slow reaction on restructuring and failures. Example of pro-active algorithm is Wireless Routing Protocol (WRP) [7] which uses an enhanced version of the distance-vector routing protocol that uses the classical Bellman-Ford algorithm [9] to calculate paths. Reactive (on-demand) routing protocols finds a route on demand by flooding the network with route request packets. The main disadvantages of such algorithms are high latency time in route finding and excessive flooding can lead to network clogging. Examples of reactive algorithms are Dynamic Source Routing (DSR)[7] and Ad-hoc On-demand Distance Vector (AODV)[6]. AODV is designed for networks with tens to thousands of mobile nodes. One feature of AODV is the use of a destination sequence number for each routing table entry. The sequence number is created by the destination node. The sequence number included in a route request or route reply is sent to requesting nodes. Sequence numbers are very important because they ensure freedom from loops and are simple to program. Sequence numbers are used by other nodes to determine the freshness of routing information. If a node has the choice between two routes to a destination, a node is required to select the one with the greatest sequence number. In AODV, every node has a routing table. In AODV, when a source node needs a connection to a destination, it broadcasts a route request which is forwarded by all other nodes in the path. Intermediate nodes record the node from which they have received this request which creates an explosion of temporary routes back to the source node. When an intermediate node receives such a message and already has a route to the desired node, it sends a message backwards through a temporary route to the requesting node. The source node then begins using the route that has the least number of hops through other nodes. It's entries are destination IP address, prefix size, destination sequence number, next hop IP address, lifetime (expiration or deletion time of the route), hop count (number of hops to reach the destination), network interface, other state and routing flags (e.g. valid, invalid). Route Requests (RREQs), Route Replies (RREPs) and Route Errors (RERRs) are message types defined in AODV. When a link fails in AODV, a routing error is passed back to a transmitting node, and the process repeats.

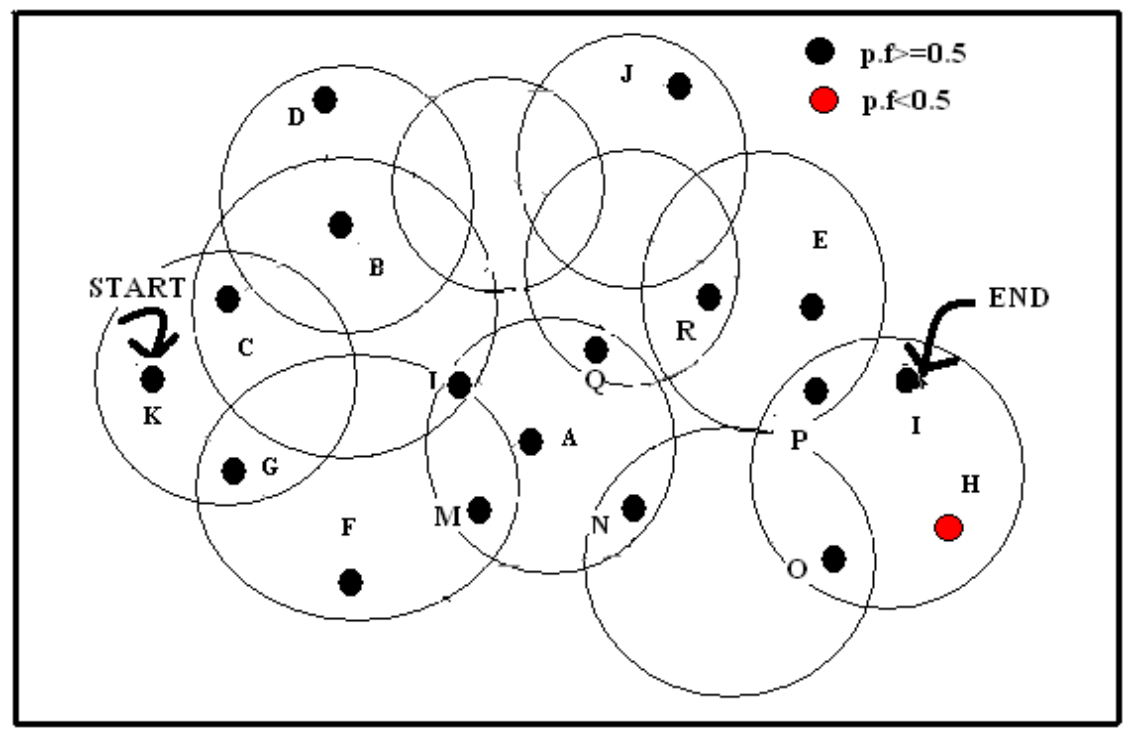

Figure 1. Topology of nodes in an Ad-hoc network 
International Journal of Wireless \& Mobile Networks (IJWMN) Vol. 3, No. 4, August 2011

Due to the dynamic changes of network topology in MANETs, however, such a protocol must constantly acquire new information to exactly reflect the network topology up-to-date, which will trigger a high overhead, especially in the presence of high mobility and large number of nodes. Also, it significantly limits its practicability $[6,9]$. Therefore, the available deterministic protocols can carry out the routing process only under a group of restricted constraints on the network coverage area size, node density or mobility. Probabilistic protocols, on the other hand, approximately predict the network topology using only probabilistic and traceable information $[11,16]$. Therefore, they can significantly reduce the amount of overhead in comparison with the deterministic protocols and thus are more scalable and topology independent.

In this paper, we propose an enhanced probabilistic routing protocol (PAODV) by introducing a queuing model for infinite packet arrivals at a node, such that the new protocol can work efficiently under different network characteristics like node density, coverage area size, and node mobility. Although we choose PAODV routing protocol to guarantee node mobility, resiliency and low latency, the basic idea of this new method can be applied to other probabilistic gossip-based routing protocols. Mathematical and extensive simulation analyses have been performed to verify the efficiency of the enhanced routing scheme in coping with different network sizes and topologies as well as node mobility. Each experiment was inspected in terms of packet delivery probability, packet drop, and throughput which have been reported in later sections.

The remainder of the paper is organized as follows: Section 2 reviews the related research in MANET routing algorithms. In Section 3, we discuss assumptions and the proposed algorithms. In Section 4, we present the simulation run. Section 5 analyzes the results obtained from simulation runs. Finally, Section 6 concludes the paper and presents the future work.

\section{RELATED WORK}

Yassein et al. [29] proposed flooding which is static routing protocol which does not need any information about the network topology to deliver packets from the source to the destination. When a node wants to send a packet, it transmits this packet to all its neighbors. Then each node that receives this packet for the first time retransmits it to all neighbors except the neighbor from which it has received which is continued until the packet reaches all nodes in the network. Each packet has a unique identifier that consists of the source address, a special sequence number used to prevent sending duplicate packets from the same node, and the destination address. The main disadvantage of flooding is the consumption of the network resources because of the high traffic load it generates. On the other hand, it ensures that the packet reaches the desired destination and gives a high packet delivery ratio.

Reactive routing schemes were developed to reduce routing overhead in mobile ad-hoc networks. Today, reactive (or on demand) routing protocols have become synonymous with the flooding of route requests (RREQs) when a path needs to be established. While this approach may be the fastest solution in a network that is not bandwidth-limited, it leads to the broadcast storm problem as identified by $\mathrm{Ni}$ et al. [34], especially in volatile routing environments. This inefficiency has been identified by many researchers in the past, and several optimizations over this blind flooding have been proposed. These approaches include the use of an expanding ring search, the use of heuristics based on connected dominating sets to reduce the number of nodes retransmitting the packets [35], the use of geographical information to direct the flooding [36] and probabilistically reducing the number of retransmissions [34]. 
International Journal of Wireless \& Mobile Networks (IJWMN) Vol. 3, No. 4, August 2011

There are many protocols that implement solutions to the flooding problem in on-demand routing protocols by enhancing route recovery mechanisms [30][31]. In general, routing protocols in MANETs can be classified into deterministic and probabilistic protocols. Some deterministic approaches try to enhance the existing deterministic protocols to get more scalable algorithms. SMORT [17] is a scalable deterministic routing algorithm that exploits secondary paths to recover from broken paths, and thus reduces the overhead produced in route recovery procedure of AODV protocol. In presence of only a few sessions, this protocol provides good scalability for different sizes of the network by adopting fail-safe multiple paths. Ad-hoc On-demand Stability Vector (AOSV) routing protocol [20], is proposed to properly and effectively discover stable routes with high data throughput and long lifetime by considering the radio propagation effect on signal strength. Some also use Global Positioning System (GPS) [21] to improve the AODV by calculating reliable distance. In [22], authors have proposed a stable, weight-based, on-demand routing protocol. While the proposed scheme may combat against link breaks due to mobility, link breaks due to the draining node energy is a factor that also must be accounted for when computing weights for stable routing.

In AODV with Backup Routing (AODV-BR) [31], nodes overhear route reply messages of their neighbors to create their own alternate routes to destination. When a node detects a broken route, it broadcasts the packet to its neighbors hopefully that one of them has a valid route to the destination and at the same time sends a RERR message to the source to initiate a route rediscovery. The reason for reconstructing a new route instead of continuously using the alternate path is to build a fresh and optimal route that reflects the current status of the network. AODV-BR concentrates on increasing route reliability by decreasing packet drop rates but it suffers from two main problems: stale routes and duplicate packet transmission. An improvement of AODV protocol based on backup route (AODV-BR) in mobile ad hoc networks was proposed by Lee et al. [25]. AODV-BR establishes the mesh and multi-paths to the destination. In AODV-BR, the primary route and alternate routes together establish a mesh structure that look similar to a fish bone. AODV-BR increased PDR but, has longer end-to-end delay.

In Neighborhood-aware Source Routing (NSR) protocol [32], each node has a partial topology that covers in addition to the 2-hop neighborhood, the links in requested paths to destinations. Link state information is maintained by broadcasting periodic HELLO messages. In case of route failure, an intermediate node tries to repair the route if either the link to the next hop has failed or the link headed by the next hop on the path to be traversed has failed. RERR message is propagated to the source node if an intermediate node uses a completely new route to destination or it has no alternate route to destination. HELLO messages in NSR incur excessive overhead to maintain the partial topology of the network. Additionally, stale route problem may affect the performance of NSR.

In AODV with Backup Routing (AODV-BR) [31], nodes overhear route reply messages of their neighbors to create their own alternate routes to the destination. When a node detects a broken route, it broadcasts the packet to its neighbors hoping that one of them has a valid route to the destination and at the same time sends a RERR message to the source to initiate a route rediscovery. The reason for reconstructing a new route instead of continuously using the alternate path is to build a fresh and optimal route that reflects the current status of the network. AODV-BR concentrates on increasing route reliability by decreasing packet drop rates but it suffers from two main problems: stale routes and duplicate packet transmission. 
International Journal of Wireless \& Mobile Networks (IJWMN) Vol. 3, No. 4, August 2011

Jian et al. [26] proposed an improvement on AODV based on reliable delivery (AODV-RD) in mobile Ad-hoc networks. In AODV-RD, a link failure fore-warning mechanism, metric of alternate node in order to better select, and also repairing action after primary route breaks is performed. AODV-RD is an improvement in AODV-BR. In this work, link failure prediction mechanism is used for checking the strength of the packet signal [27]. If the strength is in warning state then an alternate route is discovered. If more than one alternate paths are present with same hop count then the route is selected whose communicating power is stronger. AODV-RD significantly increased packet delivery ratio (PDR).

Sethi et al. [28] proposed an Optimized Reliable Ad-hoc On-Demand Distance Vector (ORAODV) protocol that offers quick adoption to dynamic link conditions, low processing and low network utilization in Ad-hoc networks. They used a mechanism of retransmission of undelivered data packets with blocking technique to enable optimal path routing and fast route delivery with an improvement of PDR.

Yassein et al. [33] proposed a Smart Probabilistic Broadcasting (SPB) scheme as a new probabilistic method to improve the performance of existing on-demand routing protocols by reducing the RREQ overhead during the rout discovery operation. The simulation results showed that the combination of AODV and a suitable probabilistic route discovery can reduce the average end-to-end delay as well as overhead, while achieving low normalized routing load, compared to AODV that uses fixed probability and blind flooding.

In [23], authors presented efficient broadcasting schemes that combine the advantages of pure probabilistic and counter-based schemes. The re-broadcast decision depends on both fixed counter threshold and forwarding probability values. The value of probability is set according to packet counter that is not equal to the number of node neighbors. In [24], the authors introduced a technique to reduce the RREQ overhead during route discovery operation, using the previous path. They argue that when the path between source and destination is changed, the new path between them will not be extremely different from the previous one. Beraldi et al. [10] presented a preliminary idea of hint-based routing for Ad-Hoc networks exploiting the duration of time passed since the last time nodes encountered with the destination, namely the encounter age. Zone Routing Protocol (ZRP) [5] is another technique that was proposed to reduce RREQ control packets, which uses a combination of two protocols, namely proactive and reactive; it takes the advantages of both protocols in order to solve the flooding of RREQ control packets.

\section{Proposed Algorithm}

From the findings of the birth and death process in queuing model [8], we can relate our proposed algorithm to the First Come First Serve (FCFS) model. The arriving packets can be regarded as the arriving customers in an unbounded queue and the route finding algorithm as the service provided to these customers (packets). According to the FCFS model, the traffic arrives randomly, which can be treated as a Poisson distribution, and are served in FCFS basis, which is an exponential distribution. Thus the probability that there will exist ' $n$ ' or greater than ' $n$ ' number of packets at a given time in a system is given by:

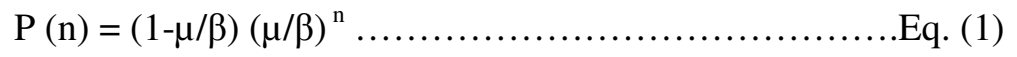

Where, ' $\mu$ ' is the arrival rate of the packets at a node, ' $\beta$ ' is the service rate of a node, and ' $n$ ' is the total number of nodes the packet has travelled, before the next node. 
International Journal of Wireless \& Mobile Networks (IJWMN) Vol. 3, No. 4, August 2011

Thus in order to decide whether a packet at a node should be forwarded to another node on a particular route is decided by considering the strength of that route, which in turn in decided by the probability function described above in equation 1 . Greater the strength of a particular route less will be the chances of packets being lost or dropped as that route is being continuously used to serve the packets for some period of time without any jitters.

The working of the proposed protocol is explained through the following example. Consider the topology of the MANET as shown in figure 1. Let the source and the destination nodes be K and I respectively. Let node $\mathrm{H}$ moves out from its current position, and the probability factor (p.f) of node $\mathrm{H}$ be less than 0.5 . Probability of each node is computed using equation 1 . In the route discovery phase, node $\mathrm{K}$ broadcasts RREQ messages to node $\mathrm{C}$ and node $\mathrm{G}$. Now, node $\mathrm{C}$ and node $\mathrm{G}$ will broadcast RREQ messages to node $\mathrm{B}$ and node $\mathrm{F}$. To the HELLO messages from node B and F, only nodes M, L and D. But the node D has a broken link, so it won't broadcast further. In the similar manner, the RREQ from node $\mathrm{M}$ and node $\mathrm{L}$ will reach node $\mathrm{A}$. Node $\mathrm{A}$ will further broadcast to $\mathrm{Q}$ and $\mathrm{N}$ which further broadcast to node $\mathrm{R}$ and $\mathrm{O}$ respectively.Node $\mathrm{O}$ broadcast to node $\mathrm{H}$ and node $\mathrm{R}$ will broadcast to E.Node $\mathrm{H}$ would not broadcast as it's probability is less than p.f. . Node $\mathrm{E}$ will broadcast to $\mathrm{P}$ which will broadcast to I,which is our final destination. RREPs(route reply) will go through the shortest path from node $\mathrm{I}$ to node $\mathrm{P}$ to node $\mathrm{E}$ and than finding the shortest path from node $\mathrm{E}$ to node $\mathrm{K}$ through node $\mathrm{Q}$, node $\mathrm{A}$,node $\mathrm{L}$, node $\mathrm{B}$,node $\mathrm{C}$. Without the proposed approach, AODV would have selected the path as $\mathrm{K}->\mathrm{G}->\mathrm{F}->\mathrm{M}->\mathrm{A}->\mathrm{N}->\mathrm{O}->\mathrm{H}->\mathrm{I}$ (as it is the shortest path). As shown in figure 2, node $\mathrm{H}$ moves out from the network topology, so there is no path from $\mathrm{H}$ to $\mathrm{I}$ and hence route discovery step is again carried out which is not the case in the proposed algorithm. Proposed approach takes care of the probability of node $\mathrm{H}$ moving out of the range of node $\mathrm{F}$ which in turn would have resulted in packet drop and finding a new path again. This in turn would have increased the network traffic in AODV which is avoided in the proposed approach.

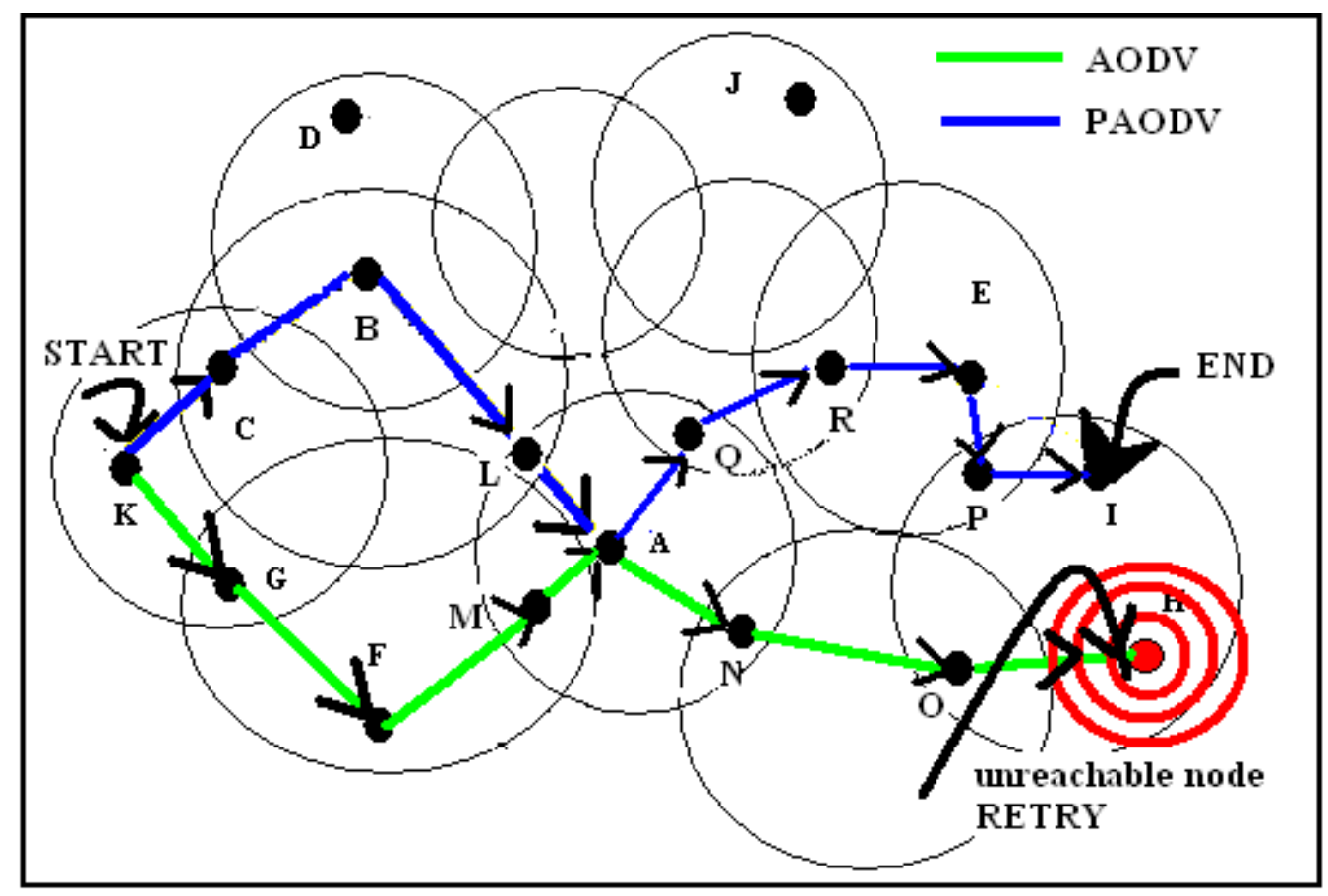

Figure 2. An example path using PAODV 
International Journal of Wireless \& Mobile Networks (IJWMN) Vol. 3, No. 4, August 2011

In this algorithm, if the probability of a route is less than the threshold value p.f (which we have assumed to be 0.5 i.e. $50 \%$ stronger route), then another route for forwarding the path is determined. The arrival of packets is calculated by the difference between current time and time when the previous packet arrived. The service time is assumed to be a constant rate of 0.001 seconds per packet. Figure 3 shows the flow chart of the proposed algorithm. Comparison of performance evaluation of AODV [6] and the proposed algorithm PAODV is shown in Table 1 where ' $n$ ' is the number of nodes in the network and ' $d$ ' is the network diameter. Complexity of AODV is obtained from the work on routing protocols for ad-hoc networks [3].

Table 1. PERFORMANCE EVALUATION

\begin{tabular}{|l|c|c|}
\hline \multicolumn{1}{|c|}{ PERFORMANCE PARAMETERS } & AODV & PAODV \\
\hline Time Complexity (initialization) & $\mathrm{O}(2 \mathrm{~d})$ & $\mathrm{O}(2 \mathrm{~d})$ \\
\hline Time Complexity (post failure) & $\mathrm{O}(2 \mathrm{~d})$ & $\leq \mathrm{O}(2 \mathrm{~d})$ \\
\hline Communication Complexity (initialization) & $\mathrm{O}(2 \mathrm{n})$ & $\mathrm{O}(2 \mathrm{n})$ \\
\hline Communication Complexity (post failure) & $\mathrm{O}(2 \mathrm{n})$ & $\leq \mathrm{O}(2 \mathrm{n})$ \\
\hline
\end{tabular}

\begin{tabular}{|c|c|}
\hline $\begin{array}{l}\text { Algorithm 1: Finding different paths } \\
\text { based on probability }\end{array}$ & Algorithm 2: Neighbor availability check \\
\hline 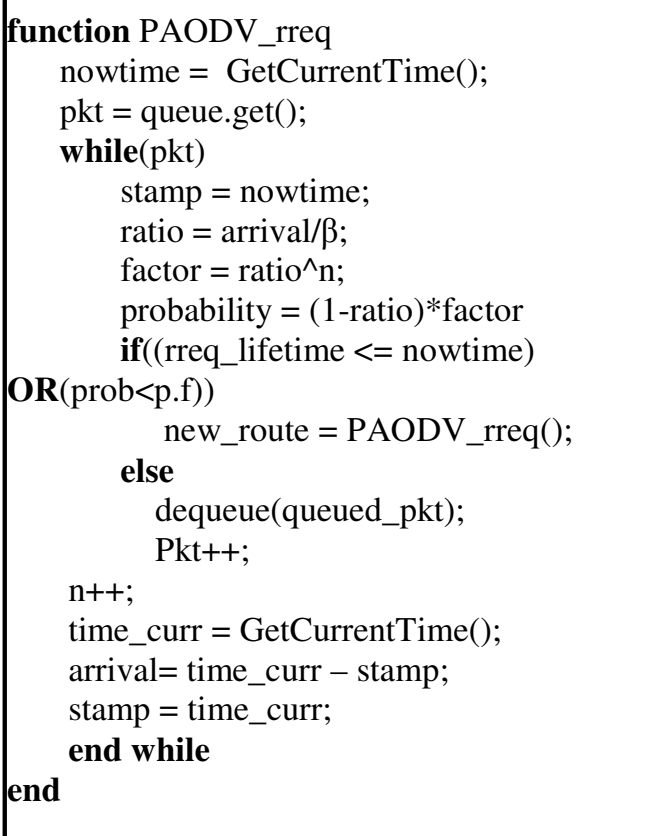 & 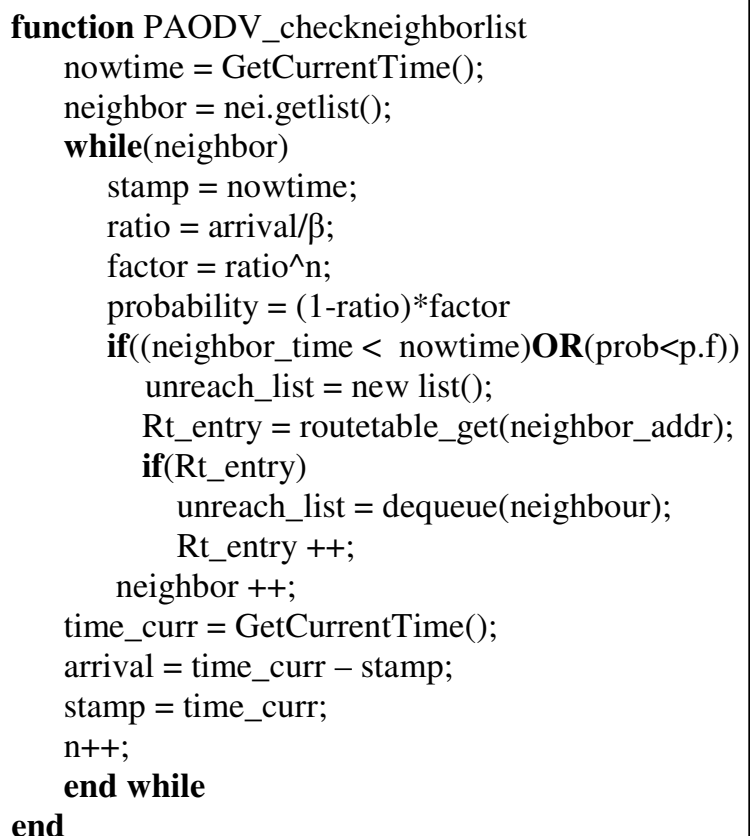 \\
\hline
\end{tabular}

The notations (variables) used in Algorithms 1 and 2 are described as below: ' $\mathrm{n}$ ' is the number of nodes visited, 'arrival' is the arrival rate of packets, 'ratio' is equal to (arrival rate $/ \beta$ ), and ' $\beta$ ' is the service rate which is equal to 0.001 . 


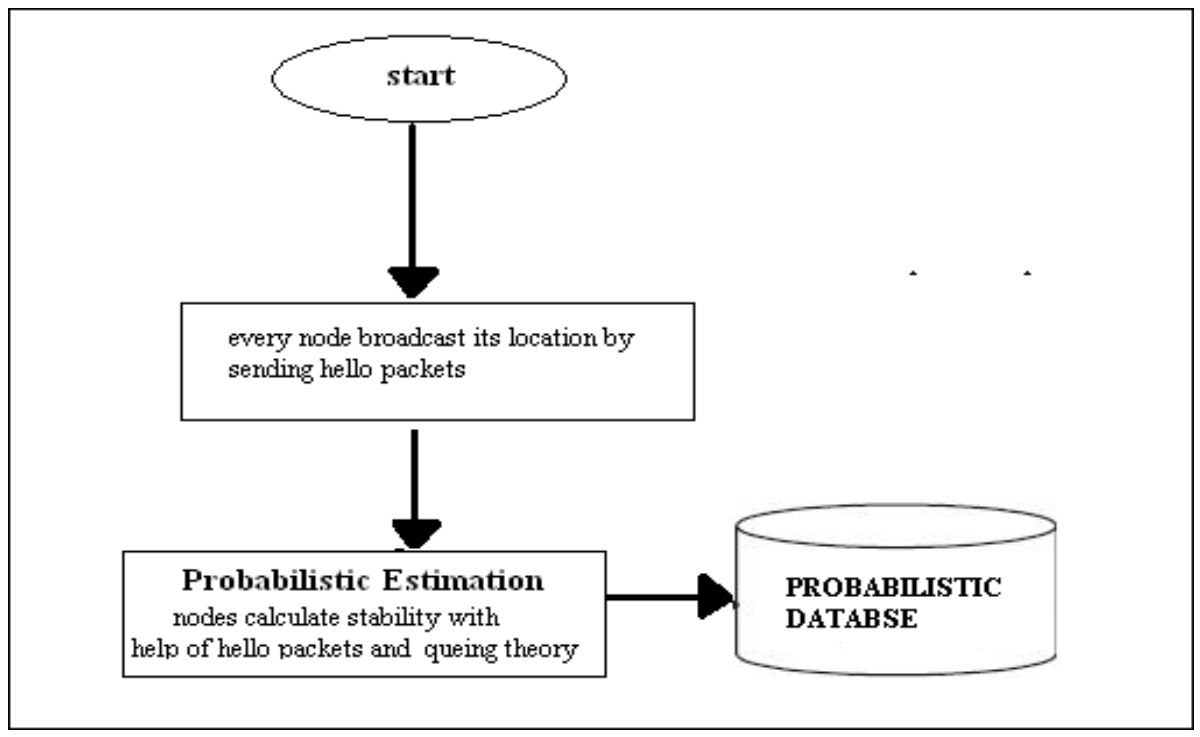

Figure 3a. Flowchart for computing probability of node stability

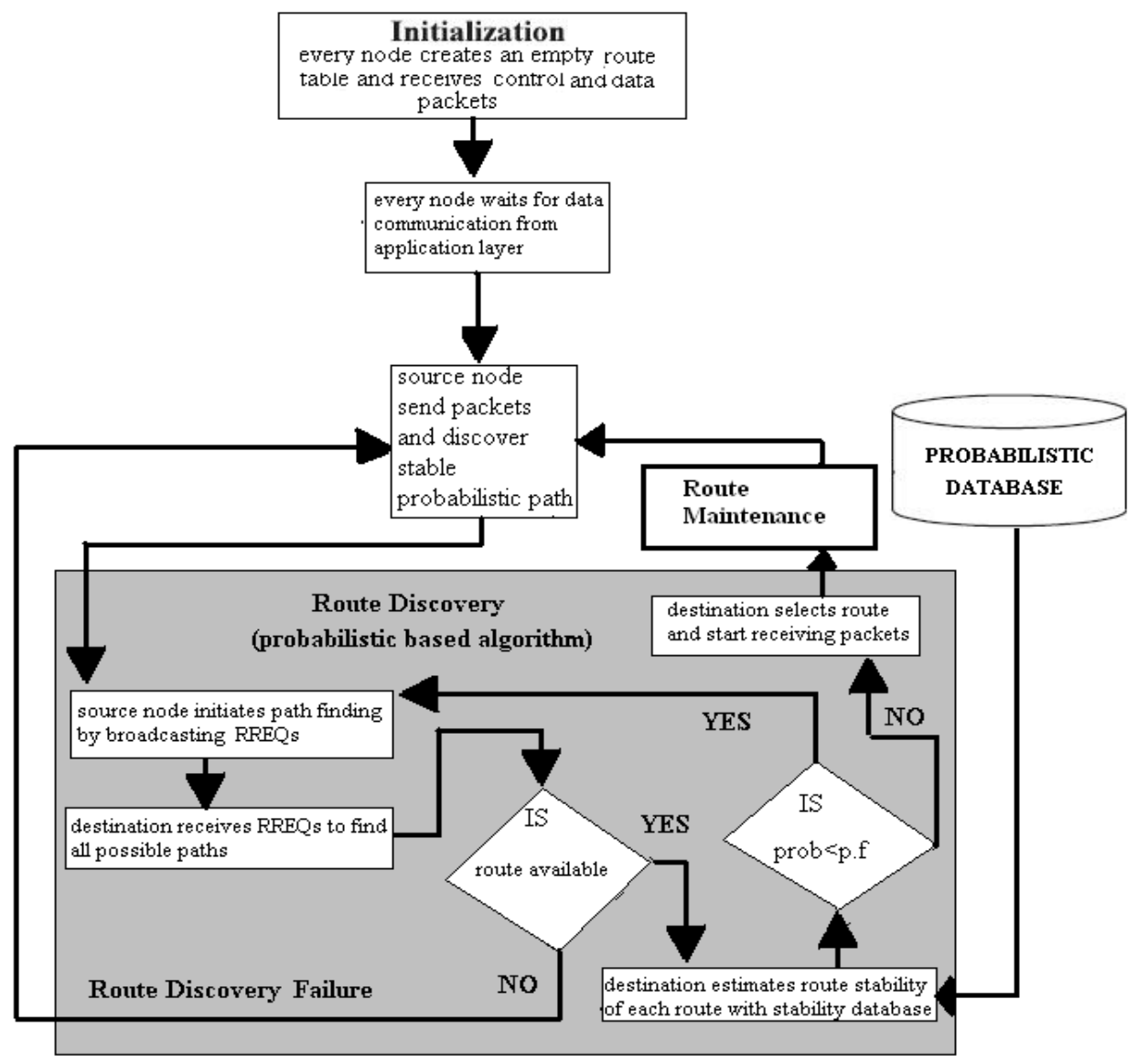

Figure 3b. Flowchart of proposed PAODV algorithm 


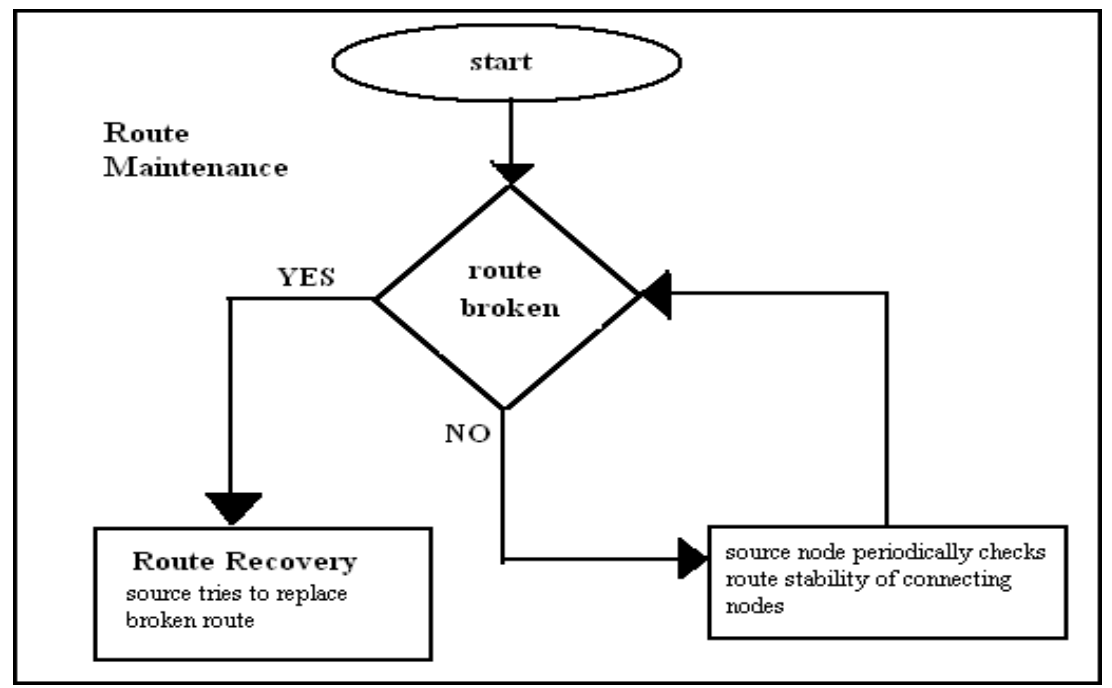

Figure 3c. Route maintenance in PAODV algorithm

Flowchart of the proposed PAODV is shown in figure 3a-3c. Figure $3 \mathrm{a}$ shows the flowchart of probabilistic database in which probabilistic estimation is made, which is used to compute the stability of the route as shown in figure $3 \mathrm{~b}$. Figure $3 \mathrm{~b}$ shows route discovery phase of the proposed algorithm. In route discovery phase, route for packet transfer is computed using probabilistic database. Figure $3 \mathrm{c}$ shows route maintenance phase of the proposed PAODV. In this phase, it is checked whether the link is stable or broken, at regular interval of time.

\section{SIMULATION}

The simulation of the proposed approach was carried out in machines running Fedora 12 with Nctuns-6.0 as the network simulator $[18,19]$. The simulation parameters are summarized in Table 2 as below:

Table 2. SIMULATION PARAMETERS

\begin{tabular}{|l|l|}
\hline Simulation time & 80 units \\
\hline Phy-model & IEEE $802.11(\mathrm{~b})$ \\
\hline nodes & $25,100,150$ \\
\hline Node movement & Randomly generated \\
\hline speed & $10 \mathrm{~m} / \mathrm{s}$ \\
\hline
\end{tabular}

NCTUns $[18,19]$ uses a distributed architecture to support remote and concurrent simulations. It uses open-system architecture to enable protocol modules to be added to the simulator. This task can be easily done in just a few mouse clicks via its GUI operating environment. The fully integrated GUI environment enables a user to edit a network topology, configure the protocol modules of a network node, set the parameter values of a protocol module, specify mobile nodes moving paths, plot performance curves, playback animations of logged packet transfers, etc. From a network topology perspective, the GUI program can generate a simulation job description file suite. Since the GUI program uses the Internet TCP/IP sockets to communicate with other components, it can submit a job to a remote simulation machine for execution. When the simulation is finished, the simulation results and generated log files are transferred back to 
International Journal of Wireless \& Mobile Networks (IJWMN) Vol. 3, No. 4, August 2011

the GUI. The user then either examines logged data, plots performance curves, or plays back packet transfer animations. We ran our simulations of Algorithm 1 and 2 using the parameters defined in Table 2, and the results were plotted. The results were plotted against varying number of network nodes. The traversal path of each node was generated randomly by using generate random path feature of the simulator. The simulations were carried out for 80 steps of the simulation time. To get realistic results, both the AODV [6] and the proposed PAODV were simulated with number of nodes varying from 25 to 100 to 150 (with a multiple of 5), having a total of 6 simulations. The packet size was fixed for all the simulations. Initially, the nodes were placed at random positions and then moved according to the randomly generated traversal path by the simulator. The moving speed of each node was kept constant throughout the simulation which was $10 \mathrm{~m} / \mathrm{s}$. This was done to ensure fairness across different simulations. By increasing the number of nodes in the topology and changing the probability factor of random nodes, better results were observed which are plotted in figure 4 to figure 7 .

\section{RESULT ANALYSIS}

\section{A. Packet drop as a Metric}

Figure 4 shows the result of the packet drop when the simulation is done with the original AODV and with the proposed algorithm PAODV. The area under the curve gives the total amount of packet drop. The simulation is carried out for 80 seconds and for 18 nodes as shown in figure 1 . When the area is calculated (area is calculated by trapezoidal rule ) this simulation gives an improvement of about $18.6 \%$ i.e. there is $18.6 \%$ less packet drop of the PAODV algorithm as compared to the original AODV.

Figure 5 shows the simulation of 150 nodes for 80 seconds. With this simulation there is an improvement of nearly $26.8 \%$ i.e. packet drop is $26.8 \%$ less in the proposed algorithm. As the number of nodes increase in the MANET, the improvement is also observed in this metric. Also, as the time of simulation increases, the improvement in the PAODV's performance is observed.

The reason for improvement can be explained easily because in the proposed algorithm, if the probability is less than p.f, then it will find a new path in that phase only rather than finding a new path again from starting as in AODV[6], which is described in flow chart of proposed algorithm shown in figure $3 a-3 c$.

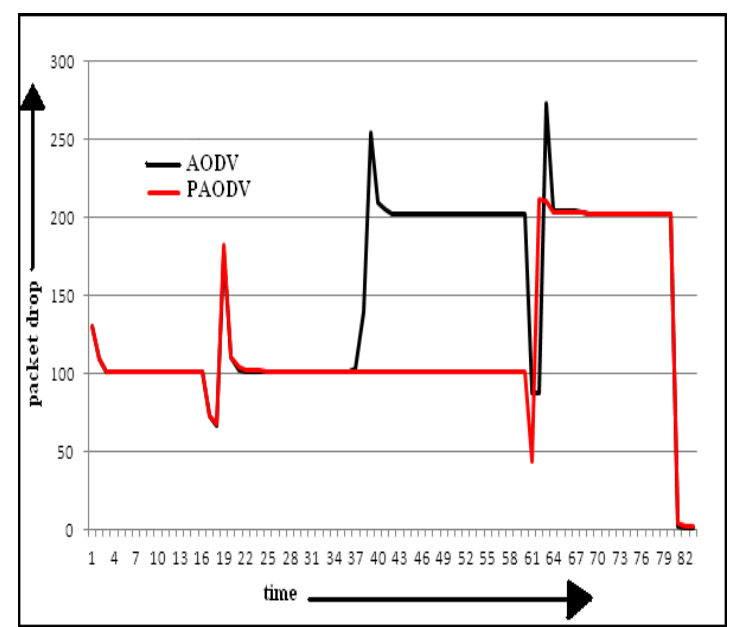

Figure 4. Time vs. number of packet drops

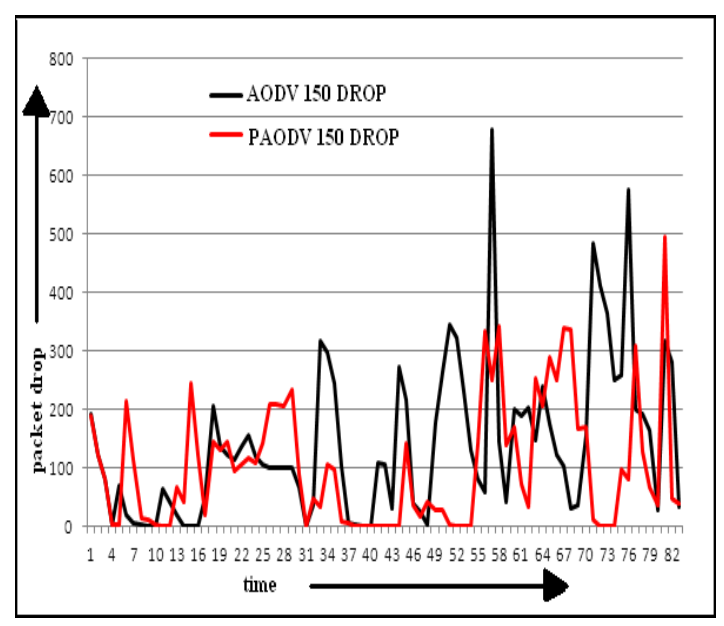

Figure 5. Time vs. number of packet drops 
As the number of nodes increase, randomness increases and the problem moves towards more realistic state. There will be more alternate paths, and hence proposed algorithm chooses best possible path based on the probability and hence performance improves drastically as shown in figure 4 and figure 5 .

\section{B. Data throughput as a Metric}

Figure 6 compares the result of the net-throughput of the original AODV with the proposed PAODV.As shown in figure 6; area under the graph of PAODV is more as compare with the original AODV. There is an improvement of nearly $3 \%$ for 25 nodes and nearly $22 \%$ for 150 nodes network.

\section{Probability factor as a Metric}

The probability factor (p.f) also changes the result improvement. A simulation of 100 nodes with proposed algorithm is carried out with p.f set to 0.8 and 0.5 . Figure 7 shows the packet drop with p.f equal to 0.8 and 0.5 .

The result shows that there is an improvement of $8.3 \%$ i.e. there is $8.3 \%$ less packet drop for p.f $=0.5$ with respect to 0.8 p.f. The p.f $=0.5$ is the optimal value and can be explained as follows:

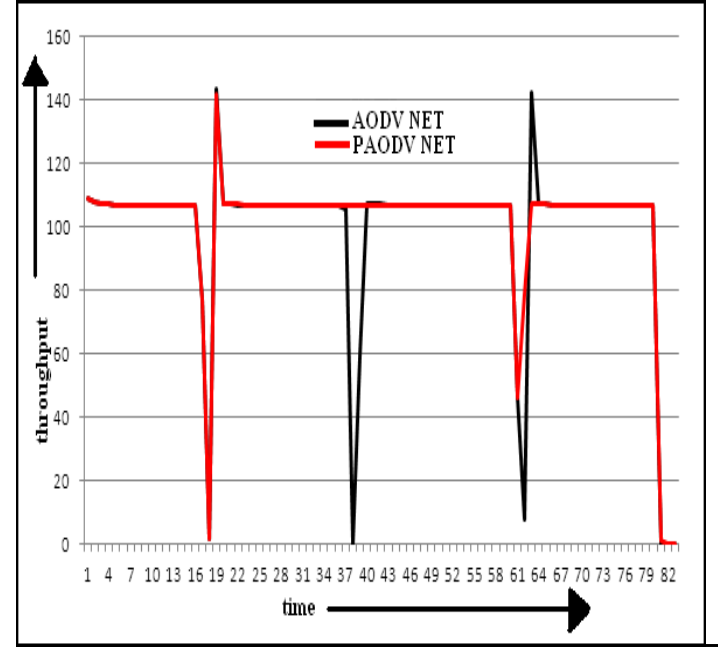

Figure 6.Time vs. throughput

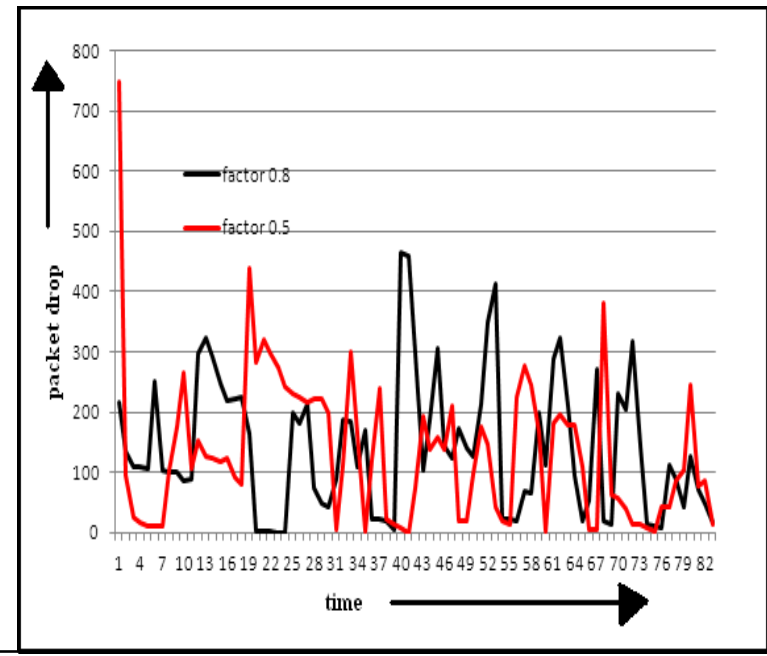

Figure 7. Time vs. no. of packets drop

Because the value (0.5) is in between 0 and 1 and the improvement in our proposed algorithm is because it prevents the route propagation time when the node won't be available after route discovery. But this is the probabilistic approach and it might be possible that the node will be available for propagation which in turn wastes the time of route discovery again. The p.f when set to 0.5 keeps track of this and hence produces optimum result in general. Also, as the topology increases, the improvement is further observed. 


\section{CONClusion AND FUture WORK}

In this paper we described a novel approach of using a probabilistic function to evaluate the packet drop in ad-hoc network. Our simulation results indicate that the packet drop reduces by around $27 \%$ for 150 node network and throughput increases by $21 \%$ for 150 node network. Therefore, we safely assume the proposed algorithm is a better approach in MANETs for adhoc on demand distance vector routing protocol(AODV) that are extensively used in the literature. It both optimizes the network performance and guarantees the communication quality. The proposed protocol provides a link failure fore-warning mechanism in the form of probability factor in order to better select an alternative route.

With simulation run of our algorithm, further properties of Ad-hoc networks may be measured. These include the in-broadcast, out-broadcast and comparisons of different nodes in the network rather than choosing some target node. As a continuation of this research in the future, we plan firstly to combine our algorithm with different routing protocols to observe, if a combined performance improvement is feasible. Secondly, we plan to study security threats along with probabilistic route finding, for secure transfer of data in MANETs. We also plan to improve upon end to end delay in data transfer.

\section{REFERENCES}

[1] C. Perkins and P. Bhagwat, "Highly dynamic destination-sequenced distance-vector routing (DSDV) for mobile computers", Proceedings of ACM SIGCOMM, pp. 234-244, (1994), London, UK.

[2] P. Jacquet, "Optimized link state routing protocol for ad hoc networks", Proceedings of IEEE International Multi Topic Conference, pp. 62-68, (2001), Lahore.

[3] Elizabeth M. Royer, C-K Toh, "A Review of Current Routing Protocols for Ad-Hoc Mobile Wireless Networks", Proceedings of IEEE PersonalCommunications-99, pp 1-5(1999), Charleston, USA.

[4] D.B. Johnson, D.A. Maltz, and J. Broch, " DSR: The dynamic source routing protocol for multi-hop wireless ad hoc networks”, Ad Hoc Networking, ed. C.E. Perkins, Addison-Wesley, (2001).

[5] Z.J. Haas, M.R. Pearlman, and P. Samar, "The zone routing protocol (ZRP) for ad hoc networks", Internet draft, Aug. (2002).

[6] C.E. Perkins, E.M. Royer, "Ad-hoc on-demand distance vector routing, in", Proceedings of the 2nd IEEE Workshop on Mobile Computing Systems and Applications, vol. 2, February, (1999), pp. 90-100, New Orleans, LA.

[7] D.B. Johnson, D.A. Maltz, "Dynamic source routing in ad hoc wireless networks", Kluwer International Series in Engineering and Computer Science (1996), pp. 153-179.

[8] Uri Yechiali, "A Queuing-Type Birth-and-Death Process Defined on a Continuous-Time Markov Chain”, Queueing Systems - Theory and Applications - QUESTA, vol. 60, no. 3-4, pp. 271-288, (2008).

[9] D. Cavendish and M. Gerla, "Internet QOS Routing using the BELLMAN-FORD ALGORITHM", Proceedings IFIP Conference on High Performance Networking, (1998), pp. 1-5, Vienna, Austria.

[10] R. Beraldi, "On message delivery through approximate information in highly dynamic mobile ad hoc networks", The Seventh International Symposium on Wireless Personal Multimedia Communications, Italy, vol. 12, (2004), pp. 15. 
International Journal of Wireless \& Mobile Networks (IJWMN) Vol. 3, No. 4, August 2011

[11] L. Rosati, M. Berioli, G. Reali, "On ant routing algorithms in ad hoc networks with critical connectivity”, Ad Hoc Networks 6 (6) (2008) ,pp. 827-859.

[12] I. Bouazizi, “ARA-the ant-colony based routing algorithm for MANETs", Proceedings of the 2002 International Conference on Parallel Processing Workshops, IEEE Computer Society Washington, DC, USA, (2002).

[13] H.F. Weddle, M. Farooq, T. Pannenbaecker, B. Vogel, C. Mueller, J. Meth, R. Jeruschkat, Bee AdHoc, "An energy efficient routing algorithm for mobile ad hoc networks inspired by bee behavior", Proceedings of the 2005 Conference on Genetic and Evolutionary Computation, ACM New York, NY, USA, (2005), pp. 153-160.

[14] G. Di Caro, F. Ducatelle, L.M. Gambardella, AntHocNet,“An adaptive nature-inspired algorithm for routing in mobile ad hoc networks", European Transactions on Telecommunications 16 (5) (2005), pp. 443.

[15] M. Roth, S. Wicker, "Performance evaluation of pheromone updates in swarm intelligent MANETs", The Sixth IFIP IEEE International Conference on Mobile and Wireless Communication Network, (2005), Atlantic City, USA.

[16] M. Roth, S. Wicker, Termite, "Ad-hoc networking with stigmergy", Global Telecommunications Conference, 2003.GLOBECOM’03.IEEE, vol. 5, (2003), San Francisco, USA.

[17] L.Reddeppa Reddy, S.V. Raghavan, "SMORT: scalable multipath on demand routing for mobile ad hoc networks", Ad Hoc Networks 5 (2) (2007), pp. 162-188.

[18] S.Y. Wang, C.L. Chou, C.H. Huang, C.C. Hwang, Z.M. Yang, C.C. Chiou, and C.C. Lin , "The Design and Implementation of the NCTUNs 1.0 Network Simulator", Computer Networks, Vol. 42, Issue 2, (June 2003), pp. 175-197 (SCI).

[19] S.Y. Wang, C.L. Chou, C.H. Huang, C.C. Hwang, Z.M. Yang, C.C. Chiou, and C.C. Lin ,NCTUns 6.0, "A Simulator for Advanced Wireless Vehicular Network Research", IEEE WiVEC 2010 (International Symposium on Wireless Vehicular Communications), (May 16-17, 2010), Taiwan, Taiwan. (Invited demo paper).

[20] J.H Tarng, B.W Chuang, F.J Wu, “A Novel Stability-Based Routing Protocol for Mobile Ad-Hoc Networks”, IEICE Transactions on Communications, (2007), E90-B(4): pp. 876-884.

[21] E.D.KanmaniRuby, S.Rajasurya, K.Swarnam, "A route stability-based optimized AODV protocol”, Proceedings RTCSP’09, Amrita Vishwa Vidyapeetham, India , pp. 1-5(2009).

[22] N. Wang and J. Chen, "A Stable On-Demand Routing Protocol for Mobile Ad Hoc Networks with Weight-Based Strategy”, IEEEPDCAT’06, pp. 166-169, (Dec. 2006).

[23] Tseng Y-C, Ni S-Y, Shih E-Y, “Adaptive approaches to relieving broadcast vol.storm in a wireless multihop mobile ad hoc network”.,IEEE Trans Comput, pp. 299-31, (2003).

[24] Castañeda R, Das SR, "Query localization techniques for on-demand routing protocols in ad hoc networks",Proceedings of the 5th annual ACM/IEEE international conference on mobile computing and networking, pp 186-194, (1999), New York, NY, USA.

[25] S.J. Lee and M. Gerla, “AODV-BR : Backup Routing in Ad hoc Networks”, In Proceedings of the IEEE Wireless Communications and Networking Conference, pp. 1311- 1316, (2000), Chicago, IL , USA. 
International Journal of Wireless \& Mobile Networks (IJWMN) Vol. 3, No. 4, August 2011

[26] LIU Jian and LI Fang-min, “An Improvement of AODV Protocol Based on Reliable Delivery in Mobile Ad hoc Networks", Fifth International Conference on Information Assurance and Security, pp. 507-510, (2009), China.

[27] Qing Li, Cong Liu, Han-Hong Jiang, "The Routing Protocol of AODV Based on Link Failure Prediction [C]", Proceeding of the International Conference on Software Process, (2008), Leipzig, Germany.

[28] S. Sethi and S. K. Udgata, " Optimized and Reliable AODV for MANET", International Journal of Computer Application, 3, No. 10, (July 2010).

[29] Muneer Bani Yassein, Sanabel Nimer, and Ahmad AL-Dubai, “The Effects of Network Density of a New Counter-based Broadcasting Scheme in Mobile Ad Hoc Networks", Proceeding of the 10th IEEE International Conference on Computer and Information Technology (CIT2010), ( 29 June - 01 July, 2010), Bradford, UK.

[30] Perkins, C. E. , Royer, E. M. and Das, “Ad Hoc On-Demand Distance Vector Routing. IETF Internet Draft.,http://www.ietf.org/internet-drafts/draft-ietf-manetaodv-03.txt, (1999).

[31] Toh, C. K., “ Associativity-based routing for ad hoc mobile networks”, Wireless Personal Communications

Journal, Special Issue on Mobile Networking \& Computing Systems, 4, 2 (March 1997), pp. $103-109$.

[32] Spohn, M. and Garcia-Luna-Aceves, “Neighborhood aware source routing”, in Proceedings of $2^{\text {nd }}$ ACM International Symposium on Mobile Ad Hoc Networking and Computing (MobiHoc),(2001), Chicago, IL, USA.

[33] Muneer Bani Yassein, Mustafa Bani Khalaf , Ahmed Y Al-Dubai, "A new probabilistic broadcasting scheme for mobile ad hoc on-demand distance vector (AODV) routed networks", The Journal of Supercomputing (2010) Volume: 53, Issue: 1, pp. 196-211.

[34] Ni, S.-Y., Tseng, Y.-C., Hen, Y. S. V , \& Sheu, J.-P. "The broadcast storm problem in mobile adhoc networks", In Proceedings of MobiCom, (2001), Rome, Italy.

[35] Spohn, M. A. "Domination in graphs in the context of mobile ad hoc networks", Ph.D. dissertation, University of California, Santa Cruz (2005).

[36] Karp, Kung, "Greedy perimeter stateless routing for wireless networks" , In Proceedings of the sixth annual ACM/IEEE international conference on mobile computing and networking, pp. 243-254, August(2000), Boston, MA, USA.

Authors

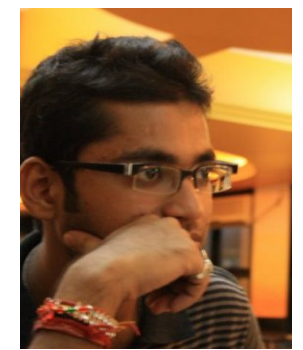

Gaurav Khandelwal is currently a fourth year undergraduate in the department of Computer Science and Information Systems at Birla Institute of Technology and Science, Pilani Hyderabad Campus, Hyderabad. He has presented his research work in Traffic Engineering in computer networks at National level technical competition held at BITS Pilani. He has also published his work on heuristic based query optimization in an International journal (IJSER). He has served as the President of Information Systems Association at BITS-Hyderabad. His current works are in mobile network security, Peer to Peer Overlays, MANETs. 


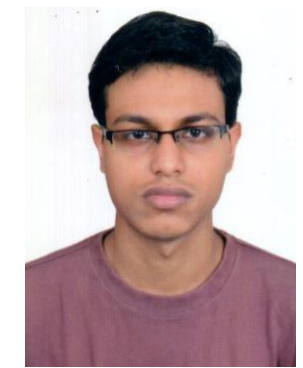

Prasanna Giridhar is currently a fourth year student in the department of Computer Science and Information Systems at Birla Institute of Technology and Science, Pilani Hyderabad Campus. At present he is doing his six months internship in the Technology and Operations Management Department of INSEAD Business School, Fontainebleau, France - working on a project to write sophisticated largescale data acquisition systems for extraction of data from multiple identified web sources and processing the raw data to extract variables of interest, with the eventual goal of developing a social network map of buyers, suppliers and firms. $\mathrm{He}$ is among top three students of Computer Science and Information Systems department and his research interests are in the areas of Peer to Peer Overlays, Mobile Ad-Hoc Networks, Database systems, Image Processing and Web Programming.

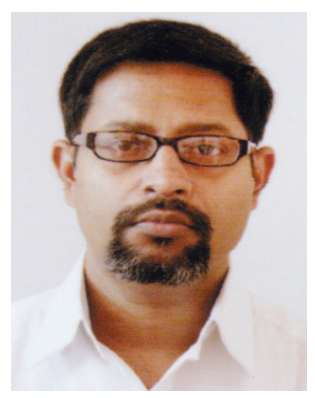

Chittaranjan Hota is Associate Professor and Head, Department of Computer Science and Information Systems at Birla Institute of Technology and Science, Pilani Hyderabad Campus, Hyderabad. He is with BITS, Pilani since the year 2000. He has worked in various Indian universities at different levels over a period of past 20 years. He has earned his Bachelor's degree in Computer Engineering, Masters degree in CSE, and $\mathrm{PhD}$ degree in CSE from Amravati, TIET (deemed) and BITS, Pilani (deemed) respectively. He had several visiting researcher and visiting professor appointments at International academic and research institutes abroad over past several years. He has published extensively in International conferences and journals. He is a life member of ISTE, and an ACM member. His research interests are in the areas of Traffic Engineering in IP Networks, Security and Quality of Service issues over the Internet, Peer-to-Peer Overlays, Mobile Ad-Hoc Networks, Cloud Computing, and Machine learning. 